\title{
How Long and What For? Tracking a Nationally Representative Sample to Quantify Internet Use
}

\author{
NOEMI FESTIC \\ MORITZ BÜCHI \\ MICHAEL LATZER \\ University of Zurich, Switzerland
}

Testing communication theories requires a valid empirical basis, yet especially for usage time measures, retrospective self-reports have shown to be biased. This study draws on a unique data set of 923 Swiss internet users who had their internet use tracked for at least 30 days on mobile and desktop devices and took part in a survey covering internet usage as well as person-level background variables. The analysis focuses on active usage time overall and on the major services Google Search, YouTube, WhatsApp, Instagram, Facebook, and the online newspaper 20 Minuten. The results showed that overall internet usage time was lower for older and higher-educated users based on both the tracking and survey data, and the reported usage time was consistently higher than the tracked usage time. The tracking data further revealed that internet users in all social groups spent the majority of their time online on a mobile device. The number of users of the major services varied mainly between age groups. These differences were less pronounced when it came to the time users spent engaging with these services. Over the course of a day, the major services varied in their frequency of use: for example, messaging peaked before noon and in the late afternoon, whereas online news use was comparably constant at a lower level.

Keywords: internet usage, tracking data, self-reports, survey, digital traces

\footnotetext{
Noemi Festic: n.festic@ikmz.uzh.ch

Date submitted: 2021-01-07

Copyright $(2021$ (Festic, Büchi, Latzer). Licensed under the Creative Commons AttributionNonCommercial-NoDerivatives 4.0 International Public License. Available at: http://journalqd.org
} 


\section{Introduction: On the Importance of Measuring Internet Usage with Tracking Data}

The way people use digital media and the internet has changed significantly in the past decade (see Latzer et al., 2020 for Switzerland). The internet is increasingly used across multiple devices, often on the go, and this use is very much integrated into everyday activities rather than being a discrete event with a clear beginning and end point. These usage habits have implications for measuring media use (for instance in terms of frequency and time), which has become more challenging as a consequence.

At the same time, theoretical questions of communication processes are increasingly addressed with sophisticated modeling techniques given the growing acknowledgement that cross-sectional regressions cannot support causal claims. However, basic descriptive knowledge about the prevalence of an empirical phenomenon that helps to contextualize specific findings and to know where to look closer in future research is often still scarce: Whether we are interested in the prevalence of filter bubbles (e.g., Dubois \& Blank, 2018), want to know what the perils of being online for adolescents are (e.g., Smahel et al., 2020), or care about internet users' privacy protection behaviors (e.g., Boerman et al., 2018), addressing these questions and advancing media and communication theories requires solid empirical evidence on internet use and a foundational understanding of the scale of use in everyday life.

Therefore, this article addresses the following research questions: How much time do people spend online and using specific services? How does this usage time differ according to sociodemographic characteristics (gender, age, education)? The results are intended to contribute descriptive knowledge that is not just intuitively interesting, but also necessary for subsequent theorizing of the causes and consequences of these observable patterns. This study relies on a combination of tracking and survey data to answer these questions. The following sections substantiate the reasons for this methodological choice. 
Internet usage data has so far mainly been collected through people self-reporting their behavior in face-to-face, phone or web-based interviews. Recently, internet use tracking has emerged as a new option to gather such data. While there are unique challenges to logging people's internet use, it is a promising and complementary new measurement approach. In addition to technological advances that have made tracking usage possible, the academic application of this new way of data collection was mainly motivated by biases in survey data, which is usually self-reported and retrospective. Although a recent metastudy attests self-reported data on media exposure a moderate reliability and a high stability (Scharkow, 2019), it is clear that answering questions like "How much time do you spend online on an average day?" is difficult and error-prone. When it comes to the use of specific services, it is likely that internet users find it even more challenging to recall exactly how many times they, for instance, scrolled through their Instagram feed and how much time they spent on the platform.

A combination of tracking data and traditional survey measures - albeit being subject to its own specific challenges (see e.g., Stier et al., 2019) - appears to be the most viable solution to circumvent methods-specific biases (see p. 4) and provide a valid description of people's everyday internet use linked with person-level background variables. Particularly given the research interest of this article-describing internet use in different social groups — including self-reported demographic and socioeconomic variables is vital (and they must be accurate). In existing big data research, such user characteristics are often inferred from user behavior such as clickstreams or consumer purchasing data (e.g., for personalized advertisements).

Such a combination of tracking and survey data has, for instance, been used to study echo-chambers in online news consumption (Cardenal et al., 2019). One of the earlier studies linking survey and tracking data (Dvir-Gvirsman et al., 2016) examined how frequently people were exposed to like-minded content and found that this occurred less often than internet users assumed. Guess et al. (2019) linked tracked Facebook sharing activity data with survey responses and demonstrate how this unique combination leads to 
the result that users share misinformation a lot less frequently than commonly assumed. Vraga and Tully (2020) demonstrated how fundamentally self-reported news exposure can differ from tracked news exposure and point to individual and contextual characteristics explaining these disparities. Aiming at measuring the implications of using recommender systems, Loecherbach and Trilling (2020) developed an online news environment that allows researchers to experiment with settings and to include user surveys. Such simulated approaches circumvent the challenge of gathering tracking data but limit external validity.

A number of studies have specifically compared self-reported and tracked internet use in terms of frequency, amount or types of use. Based on a large sample of Facebook users, Ernala et al. (2020) revealed that compared to Facebook's server log data, users significantly overestimate the time spent on the platform, while underestimating how often they access the site. For a non-representative sample of 690 Dutch internet users, Araujo et al. (2017) found that self-reported internet use time is higher in comparison with tracked data on time spent online. These differences were partially explained by both internet-use related and contextual factors. Similarly, Scharkow (2016) found that for a large random sample of internet users, the correlation between self-reported and logged internet use was low. Further, internet usage time and frequency were among those measures that were particularly overreported. These results are limited to internet use on home computers. Naab et al.'s (2019) study compared self-reported measures of internet use with results from mobile experience sampling for a sample of students. They found that the participants consistently reported spending more time on Facebook, WhatsApp and YouTube than was found through the in-situ reports that were collected for a duration of two weeks.

Especially relevant in this context are not only comparisons between usage times, but relationships with other relevant variables. For a sample of college students and MTurk workers, Jones-Jang et al. (2020) found that correlations between self-reported usage data and relevant outcomes were lower than between logged usage data and these outcomes, indicating that not only media usage (see section above), but also media usage effects may often be underestimated rather than overestimated. 
These empirical results relying on a combination of survey and tracking data suggest that given the methodological advances and changed usage habits, very basic questions on internet usage time in different social groups need to be readdressed since there are empirically founded concerns about the accuracy of self-reports. The overview of existing literature presented above reveals several research gaps concerning the quantitative description of internet usage time with survey and tracking data: there is a lack of representative data that was collected in a natural usage situation and that includes mobile use. This article employs an innovative methodological approach introduced below and aims to contribute to filling these research gaps.

\section{Method}

\section{Data Collection}

The data collection for this article consisted of two main parts: (1) All participants were already part of a mobile (smartphone or tablet) tracking panel (see p. 6 for a more detailed description of the sample). To gather tracking data for not only mobile but also desktop devices, the participants received installation instructions for a passive metering software for their desktop or laptop device at the start of the field phase. However, not all participants of the study used a desktop device or installed the passive metering software despite maybe owning such a device. Therefore, the proportion of mobile usage time may be slightly overestimated compared to the general population. Between November 2018 and January 2019, we collected tracking data through the passive metering software on private mobile and (if the participants opted in) desktop or laptop devices. The collected variables were the URL of a visited webpage (desktop and mobile) or name of a used app (mobile only), duration and time of the visit, device, and operating system used. (2) At the end of this phase, the participants were advised to uninstall the passive metering software from their desktop or laptop devices and were invited to complete a survey questionnaire. 
The survey took 30 minutes on average and included questions on personal background, internet use, risk awareness online, and various internet-use related attitudes.

The participants received a small pecuniary incentive for their participation in the tracking and survey. All participants in the tracking and survey gave informed consent on their participation and the research design was approved by the University of Zurich's ethics review board.

\section{Sample}

The independent market and social research company LINK recruited and sampled the participants from an existing internet panel. For the desktop and laptop tracking data, we relied on a passive metering solution by Wakoopa. The internet panel is actively recruited, which is important in order to reduce the likelihood of a self-selection bias where people with lower privacy concerns would be more likely to select themselves into such a sample. The initial sample of 1'202 respondents is representative by age, gender, region, household size, and employment status for Swiss internet users aged 16 and over. While the overall survey sample is representative for the Swiss internet user population, the tracking sample somewhat overrepresents middle-aged users (aged around 40) and slightly underrepresents the oldest group (70-85). Comparing the survey and tracking samples in terms of other key sociodemographic variables, all proportions were within a single percentage point difference.

The data required preprocessing before analysis. At the level of tracked events (i.e., a site visit), we removed all events with 0 seconds of usage time $\left(N_{\text {tracked events }}=233\right.$ '675) because these reflect automatic redirects and were not part of the participants' actual internet usage; the passive metering software recorded any visited URL regardless of the time spent on it. At the level of participants, we excluded those participants $\left(N_{\text {participants }}=\right.$ 51) who were tracked for fewer than the thirty days planned in the study design. Further, we excluded extreme outliers who reported more than 17 hours of internet usage per day 
for reasons of plausibility $\left(N_{\text {participants }}=2\right)$. The resulting final sample $\left(N_{\text {participants }}=923\right.$, $N_{\text {tracked events }}=13^{\prime} 252^{\prime} 235$ ) formed the basis for the reported results in this article.

\section{Measures}

The analyses in this article rely on a combination of the tracking (usage time for the internet and major services) and the survey data (self-reported use for the internet in total and major services, demographic and socioeconomic variables).

Self-reported usage time (survey). We asked the respondents to assess their overall usage time of the internet by answering the following question: "For how many hours in total do you use the internet on an average day? Please think of all your internet use (at home, at work, on the road, etc.). Please give us the number in hours, e.g., 15 minutes $=0.25$ hours."

Self-reported usage of major services (survey). The respondents were asked to indicate which of the following services they used at least occasionally (multiple responses possible): Google Search, YouTube, WhatsApp, 20 Minuten (most popular free online newspaper in Switzerland), Facebook and Instagram.

Social background variables (survey). Since the goal of this article is to compare internet usage in different social groups, the survey included various demographic and socioeconomic variables. In particular, the respondents were asked to report their gender (female, male) as well as their age in years, which was recoded into four groups (16-29, $30-49,50-69,70-85)$. They also reported their completed levels of educational attainment, which were recoded into three levels: individuals whose highest completed education level was the compulsory school were assigned the value low and those with tertiary qualifications (university degree or similar) were assigned the value high. 
Usage time: Internet total (tracking). The passive metering software logged the time the users spent on every website or app. We summed up these usage times for every participant and divided this sum by the number of days for which the respective participant's internet use was tracked (this varied between 30 and 120 days).

Usage time: Major services (tracking). The measure for the use of major services was calculated by filtering the tracking data for the occurrence of these apps and websites, and extracting these cases from the data set. Analogous to the measure for total internet usage time, we summed up these usage times for every participant who reported using the respective service in the survey and divided this sum by the number of days for which the respective participant's internet use was tracked.

It is important to note that the tracking software measured active use of applications or websites, meaning the app or browser window was in the foreground. Therefore, our use data corresponds to the time that users spent on these respective apps or websites but does not reflect, for example, the time the participants were available to receive a message or a call on WhatsApp.

\section{Data Analysis}

Data analysis relied on descriptive statistics and particularly on mean score comparisons between different social groups in $R .^{1}$

\section{Results}

The time Swiss internet users spend online every day was measured both through the tracking and the survey.

\footnotetext{
${ }^{1}$ The R script for the analysis and the detailed results are available at: https://osf.io/j5mhn/?view only=7e82e560f19945d4bbbae168cbbcde3e
} 

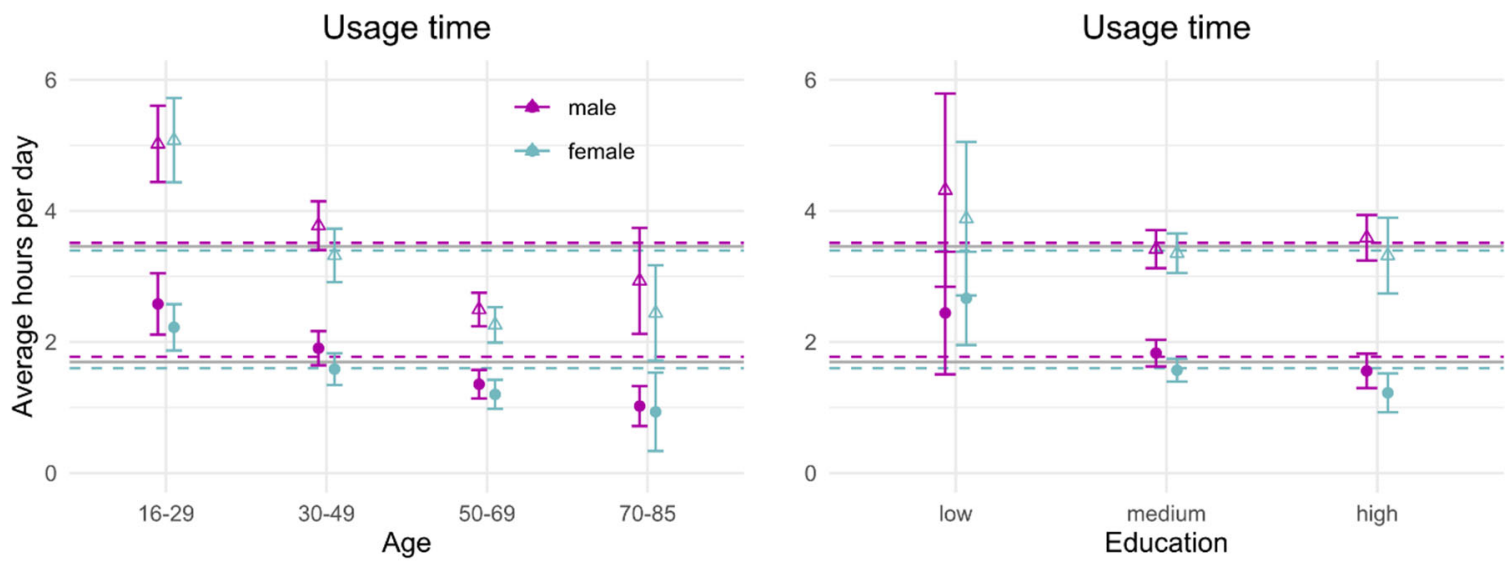

Figure 1. Total daily internet usage (desktop and mobile) by gender, age and education.

Note. Vertical bars represent 95\% confidence intervals; Horizontal lines represent overall (solid) and group means (dashed). Y-axis indicates means on a continuous scale (hours per day). Lines with points as markers are for tracking data, those with triangles for survey data. Markers are offset for visual reasons, not because they occupy a different space on the X-axis; this pertains to all other figures. $N=923$ internet users.

Figure 1 depicts differences in daily average tracked and self-reported usage time between different social groups. The results from the tracking and survey data revealed that -with some differences-younger internet users and those with lower levels of educational attainment tend to spend more time online every day. These differences were particularly pronounced for male members of the youngest age group, who spent 1 hour and 39 minutes more online on average compared to females aged 70 and over based on the tracking data. While male internet users tended to be online longer every day, these differences between the genders were not significant. The tracking data revealed that overall, Swiss internet users spent less than two hours on the internet every day. The selfreports were consistently higher in all age and educational groups and across both genders. The mean time that internet users spent online was 1.70 hours based on the tracking data and 3.45 hours based on the survey data. These measures were weakly correlated: $r(933)$ $=.24, p<.001$. 
Based on the tracking data, the majority of this total internet usage time was spent on mobile devices ( $M=1.34$ hours per day). The proportion of internet usage time that was through mobile devices tended to be lower for older individuals: while females aged 16 to 29 spent $80 \%$ of their time online on a mobile device, this proportion was only at $56 \%$ for females aged 70 and over. There were no significant differences between education groups or between the genders. Internet users across all age and education groups and across both genders spent the majority of their time online on a mobile device.
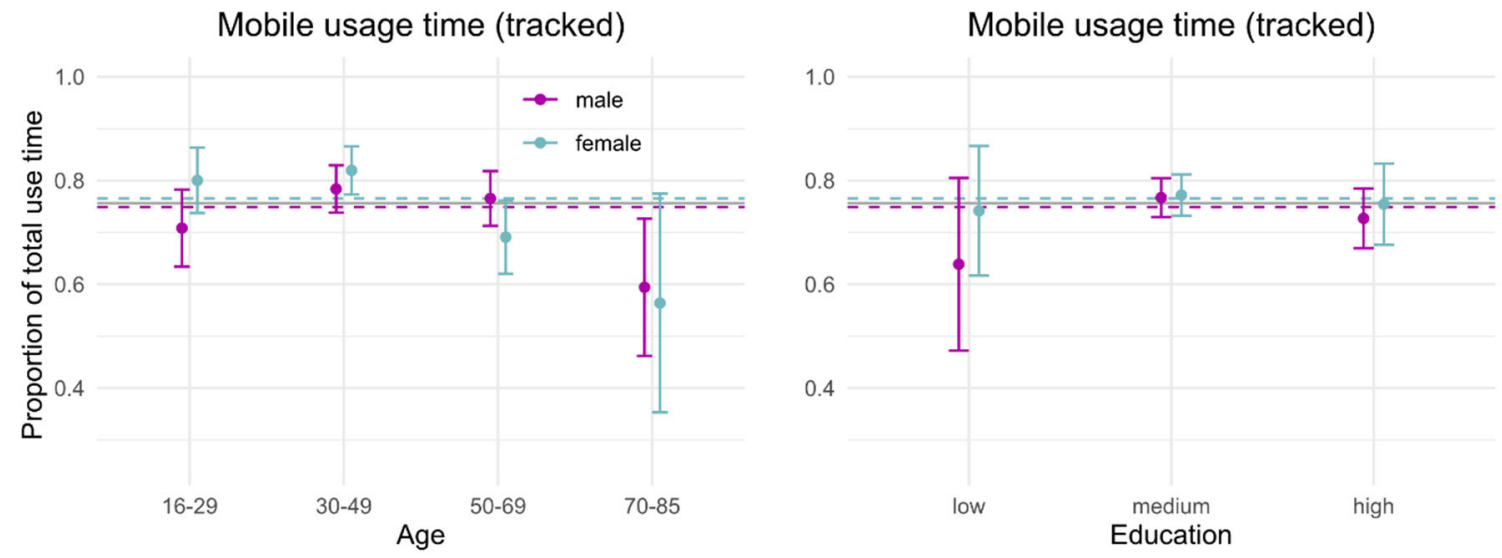

Figure 2. Mobile usage time as a proportion of total usage time by gender, age and education.

Note. Vertical bars represent 95\% confidence intervals; Horizontal lines represent overall (solid) and group means (dashed). Y-axis indicates means on a continuous scale (hours per day). $N=923$ internet users.

In addition to these global results on total internet use, this article specifically aims at empirically investigating the use of certain popular services. As the results in Table 1 reveal, virtually all Swiss internet users reported (survey) using WhatsApp and Google Search. A clear majority also used YouTube and Facebook, while 20 Minuten was used by half and Instagram was used by around a third.

These six services accounted for more than a quarter $(26.77 \%)$ of total internet use in terms of tracked events (tracking). WhatsApp was the service that Swiss internet users spent the most time using on average (messages and calls), although the variance was also 
very high. There were also differences regarding the devices with which the internet users accessed the services. While WhatsApp, Instagram and the newspaper 20 Minuten were almost exclusively used through mobile devices (92-99\% mobile accesses), the ratio between mobile and desktop accesses was more balanced for Facebook, Google Search and YouTube. The latter was the only service that was more commonly used on desktop devices.

Table 1. Descriptive overview: user groups, proportions of mobile accesses and usage times.

\begin{tabular}{lcccc}
\hline User group & $\begin{array}{c}\% \text { mobile } \\
\text { accesses }\end{array}$ & $\begin{array}{c}M \text { usage time } \\
\text { (minutes per } \\
\text { day })\end{array}$ & $\begin{array}{c}S D \text { usage time } \\
\text { (minutes per } \\
\text { day })\end{array}$ \\
\hline WhatsApp & $\begin{array}{c}97.51 \% \\
(N=900)\end{array}$ & $\begin{array}{c}98.64 \% \\
\left(N=1{ }^{\prime} 252^{\prime} 757\right)\end{array}$ & 13.23 & 34.53 \\
\hline Google Search & $\begin{array}{c}96.10 \% \\
(N=887)\end{array}$ & $\begin{array}{c}51.62 \% \\
\left(\mathrm{~N}=474^{\prime} 614\right)\end{array}$ & 3.28 & 8.65 \\
\hline YouTube & $\begin{array}{c}87.87 \% \\
(N=811)\end{array}$ & $\begin{array}{c}45.06 \% \\
(N=183 ' 510)\end{array}$ & 11.91 & 32.55 \\
\hline Facebook & $\begin{array}{c}69.34 \% \\
(N=640)\end{array}$ & $\begin{array}{c}60.81 \% \\
\left(N=353^{\prime} 720\right)\end{array}$ & 9.42 & 26.37 \\
\hline 20 Minuten & $\begin{array}{c}51.44 \% \\
(N=475)\end{array}$ & $\left.\begin{array}{c}91.92 \% \\
(N=124\end{array} 545\right)$ & 3.46 & 10.17 \\
\hline Instagram & $38.79 \%$ & $93.37 \%$ & 5.08 & 14.66 \\
& $(N=358)$ & $\left(N=219^{\prime} 499\right)$ & & \\
\hline
\end{tabular}

Note. User group depicts the share of internet users who reported using the service (survey). $\%$ mobile accesses shows the share of tracked events for the respective service that were through mobile devices. The last two columns show the mean and standard deviation for the tracked time spent on these services (tracking).

When looking at how common using these services is in the Swiss internet user population, there were particularly significant differences across age groups (see Figure 3 ). While the use of WhatsApp and Google Search was almost uniformly distributed in the Swiss internet user population, there was a slight tendency for older internet users to use these services less. The same trend was found for YouTube and — even more pronounced— 
for Facebook and Instagram. 20 Minuten was the only service that was more widespread among older age groups.

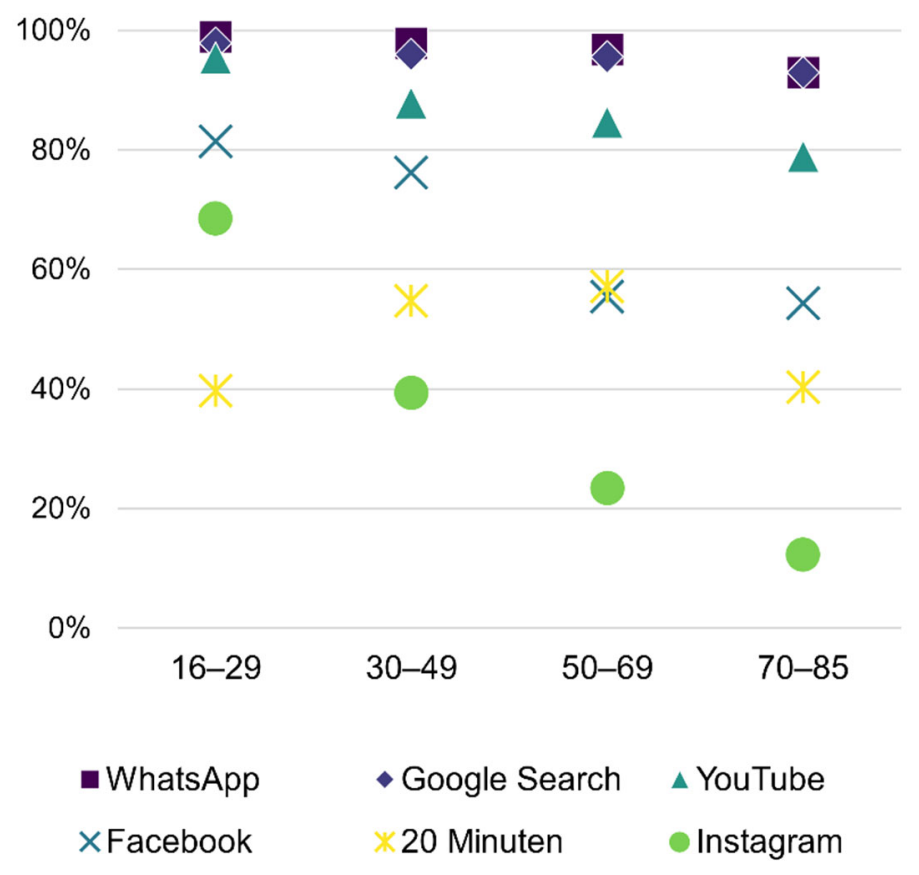

Figure 3. User group of the services by age.

Note. The percentages depict the shares of the respondents who reported using a service in the survey. $N=923$ internet users.

Further, among those internet users who reported using a certain service, we investigated whether the time spent using these services differs between age and educational groups as well as between the genders. Figure 4 shows how the daily time spent using Google Search varied between different social groups. 

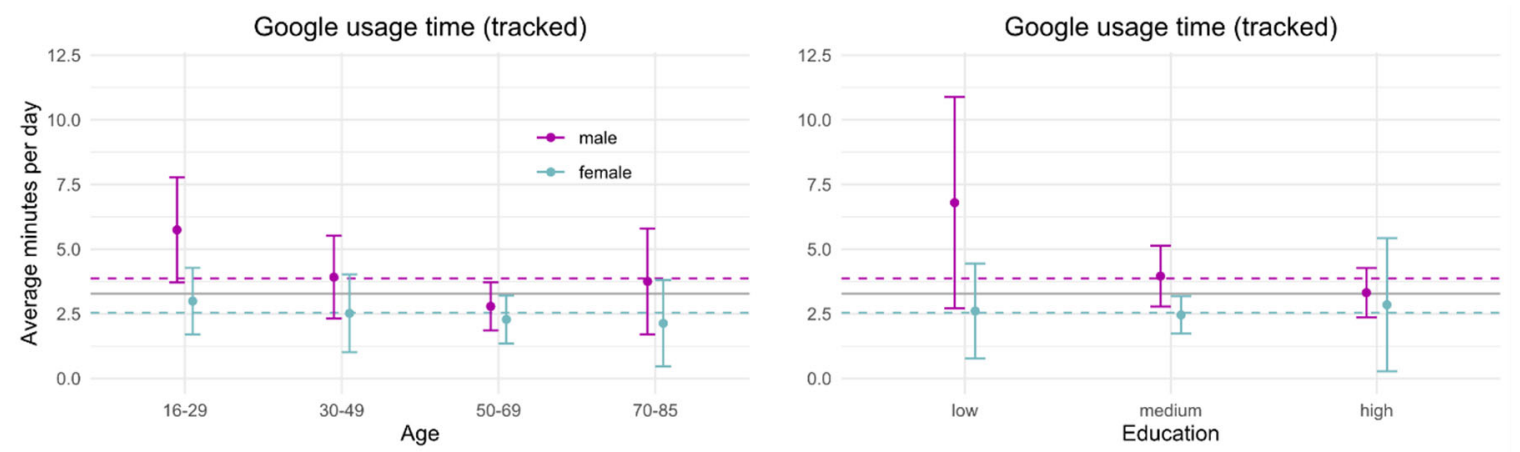

Figure 4. Daily average Google Search usage by gender, age and education.

Note. Vertical bars represent 95\% confidence intervals; Horizontal lines represent overall (solid) and group means (dashed). Y-axis indicates means on a continuous scale (minutes per day). $N=887$ Google Search users.

The relationship between age and time spent on Google Search was U-shaped, particularly for men: those aged between 50 and 69 spent less time on Google Search than the younger and older groups. Female internet users tended to spend less time on Google Search: while male internet users aged 16 to 29 spent 5.75 minutes per day on Google Search, this number was significantly lower at 2.13 minutes for females aged 50 to 69 .

There were no significant differences between the educational groups regarding the time spent on Google Search. Male internet users with a low level of educational attainment had the highest mean Google Search usage time.

Figure 5 reveals the results for the same comparisons for the online newspaper 20 Minuten. There were no significant differences between age and educational groups as well as across both genders for the time spent using 20 Minuten. 

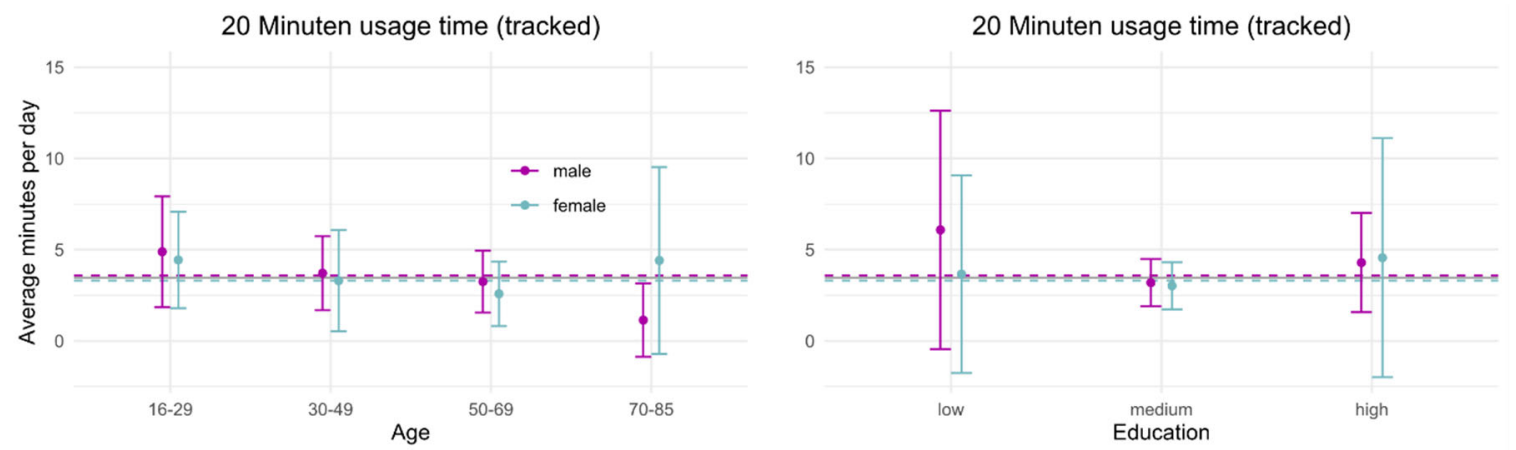

Figure 5. Daily average 20 Minuten usage by gender, age and education.

Note. Vertical bars represent 95\% confidence intervals; Horizontal lines represent overall (solid) and group means (dashed). $\mathrm{Y}$-axis indicates means on a continuous scale (minutes per day). $N=475$ users of 20 Minuten.

Figure 6 presents the differences in usage time for WhatsApp. Among female WhatsApp users, there was no difference in usage time between age and educational groups. However, male WhatsApp users in the youngest age group (16-29) used WhatsApp significantly longer every day than those aged between 50 and 69. Those aged between 16 and 29 were the only group with a significant gender difference: young male WhatsApp users spent a lot more time on the direct messaging service $(M=25.6, S D=$ 40.4) than their female peers $(M=11.6, S D=24.3)$.
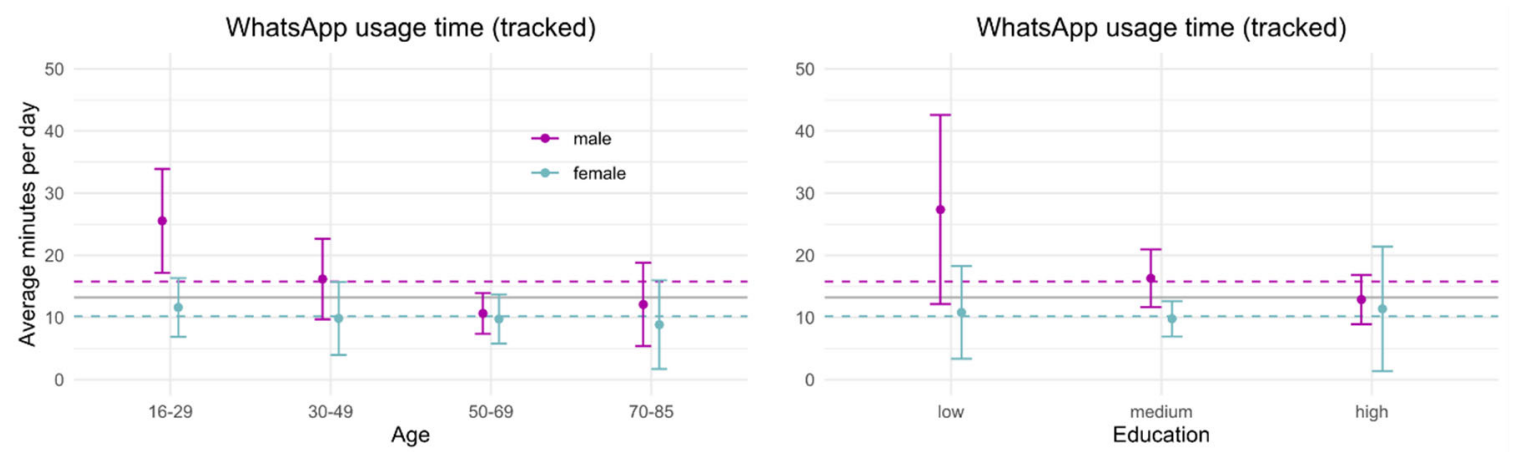

Figure 6. Daily average WhatsApp usage by gender, age and education.

Note. Vertical bars represent 95\% confidence intervals; Horizontal lines represent overall (solid) and group means (dashed). Y-axis indicates means on a continuous scale (minutes per day). $N=900$ WhatsApp users. 

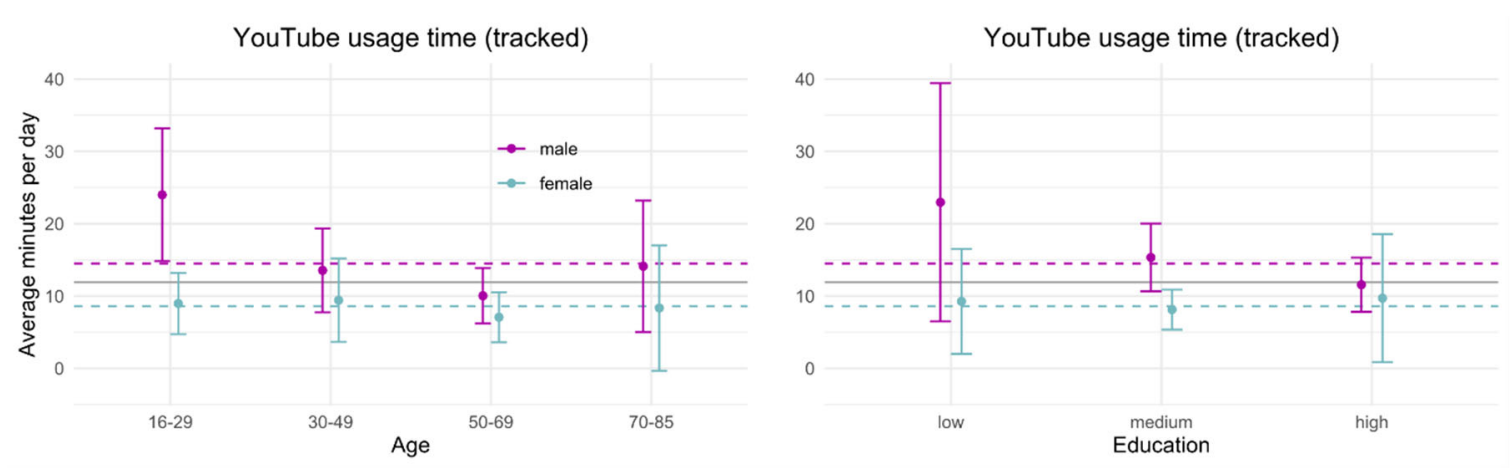

Figure 7. Daily average YouTube usage by gender, age and education.

Note. Vertical bars represent 95\% confidence intervals; Horizontal lines represent overall (solid) and group means (dashed). Y-axis indicates means on a continuous scale (minutes per day). $N=811$ YouTube users.

For YouTube usage time, Figure 7 depicts the differences between social groups. Male internet users in the youngest age group or with low educational attainment were the groups that spent the most time on YouTube (24 and 23 minutes, respectively). Among those aged 16 to 29 was the only significant difference between the genders where females spent significantly less time on YouTube. In general, time spent on YouTube decreased with age, although those aged 70 and over use YouTube longer every day than those between 30 and 69. There were no significant differences between educational groups, but especially for men, time spent on YouTube tended to be higher for those with lower educational attainment.

Figure 8 presents differences in usage time for Facebook use. For the time spent using Facebook, there were no significant differences between age and educational groups or across the genders. The time spent on Facebook tended to have a U-shaped relationship with age and male Facebook users tended to use the service longer every day. 

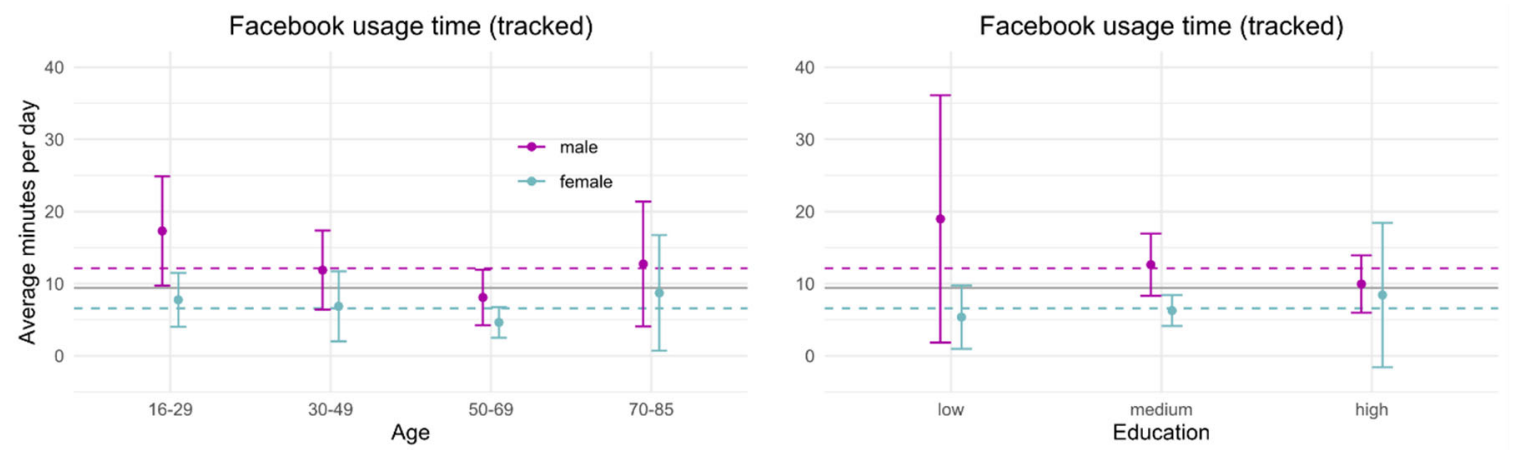

Figure 8. Daily average Facebook usage by gender, age and education.

Note. Vertical bars represent 95\% confidence intervals; Horizontal lines represent overall (solid) and group means (dashed). Y-axis indicates means on a continuous scale (minutes per day). $N=640$ Facebook users.

Figure 9 shows the mean time spent using Instagram for different groups. Males and females did not systematically differ in the time they spent using Instagram. Younger internet users generally spent more time on Instagram every day. Between educational groups, there were no significant differences. It must be noted that in the oldest age group, there were only 3 male and 4 female Instagram users in the sample. Therefore, the mean for this group should only be interpreted cautiously. For females with high levels of educational attainment, both the mean time spent using Instagram and the variation in time spent were very high $(M=8.10, S D=28.30)$.

Figure 10 depicts how many tracked events our sample contained across the course of a day. Regarding internet use in total, the amount of tracked events steadily increased from the early hours of the morning (about $6 \mathrm{am}$ ) and showed a clear peak between 4 and $5 \mathrm{pm}$. Thereafter, internet use started to decrease again. The daily usage pattern for the major services was similar in that there were less tracked events during the night and the use of these services started to increase at around 6 am in the morning. 

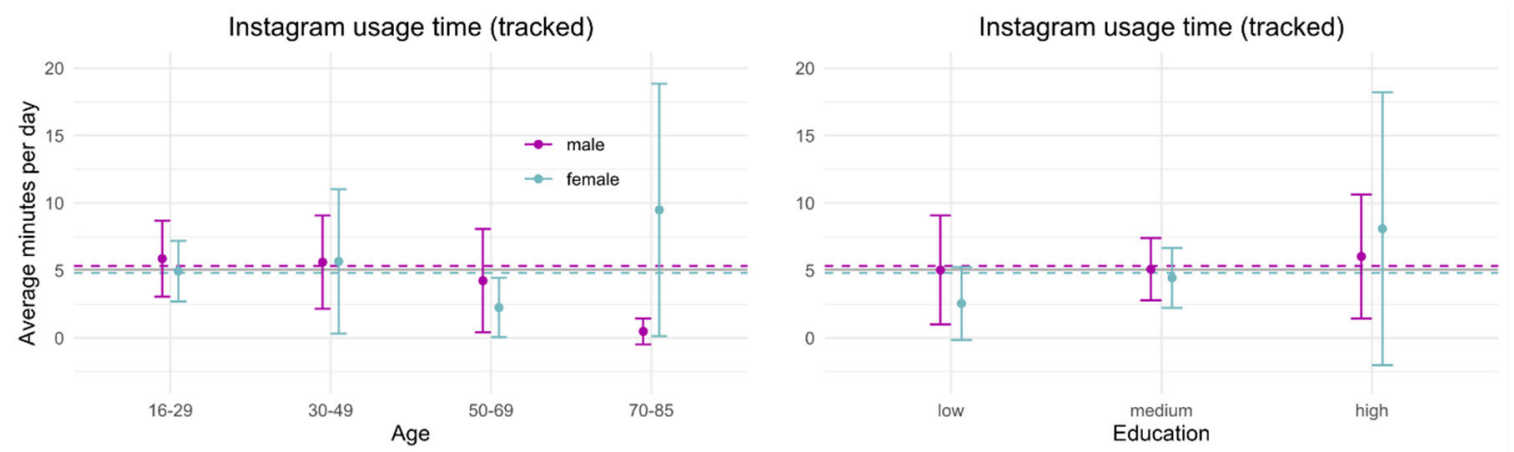

Figure 9. Daily average Instagram usage by gender, age and education.

Note. Vertical bars represent 95\% confidence intervals; Horizontal lines represent overall (solid) and group means (dashed). Y-axis indicates means on a continuous scale (minutes per day). $N=358$ Instagram users.
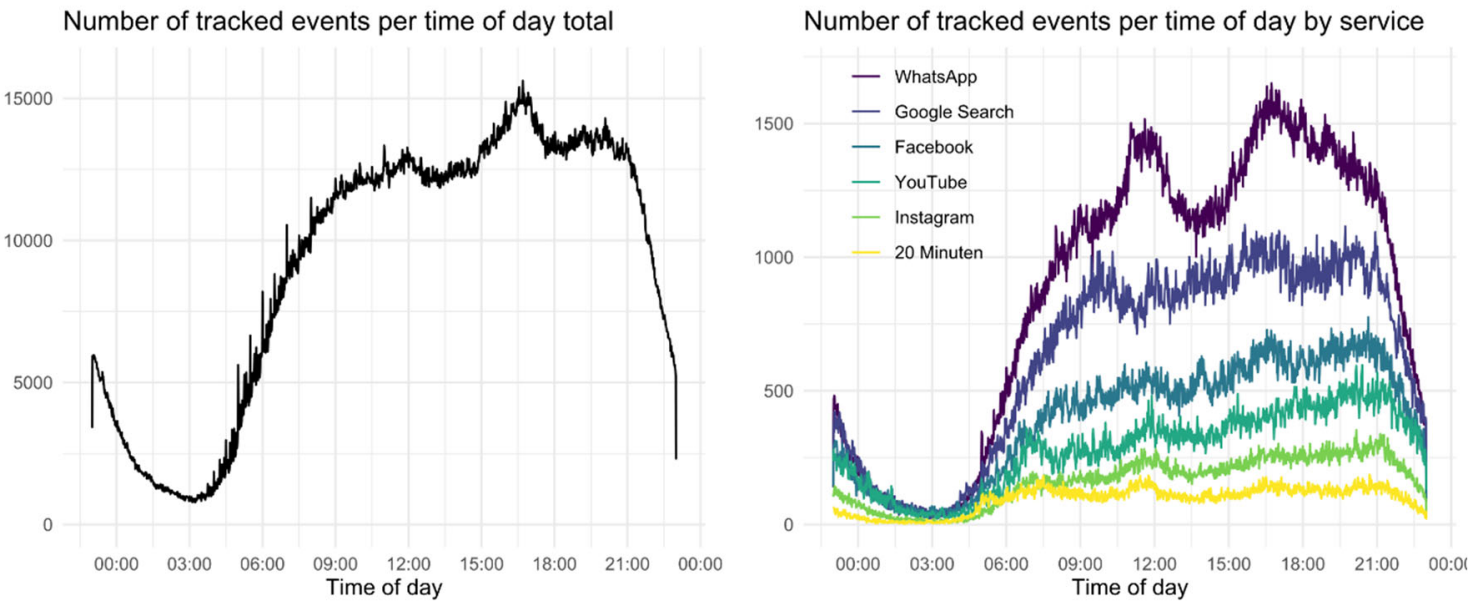

Figure 10. Number of tracked internet usage events per time of day.

Note. $N=923$ internet users for the left panel; $N=$ user groups of the respective services (see Table 1) for the right panel.

There were a few differences between the services we investigated. For WhatsApp, most uses occurred just before $12 \mathrm{pm}$ and at $5 \mathrm{pm}$, with less activity on the app in the morning and during the afternoon. Internet users used Google Search, Facebook, YouTube and Instagram most heavily in the evening (around $9 \mathrm{pm}$ ), although there were also smaller peaks in the morning (e.g., at around 7 am for YouTube). The use of the online newspaper 
20 Minuten was relatively uniformly distributed across the course of the day, except for smaller numbers of tracked events during the night.

\section{Discussion and Conclusion}

Relying on a combination of tracking and survey data, this article aimed at describing how much time internet users in different social groups (gender, age, education) spend online and using different services.

Both the tracking and survey results on usage time presented a consistent picture in terms of differences across social groups: while there were no significant differences between genders, younger people and those with lower levels of educational attainment tended to spend more time on the internet overall. While this is true for both the tracked and self-reported usage measures, we found stark differences in usage time between these two measures. Based on the tracking results, on average, people spend less than two hours a day on apps or websites, which is half the time they self-reported spending on the internet. These differences provided further indication for the importance of combining both methods for a valid empirical measurement of internet use and refinement of measurement strategies (it should not be assumed that tracking measures a ground truth and self-reports are always biased). Jürgens et al. (2019), for instance, identified sampling, selection and response biases that are specific to tracking data and conclude that "tracking data should not by default be considered an unbiased source of 'true' media exposure" (p. 612). Further, the data does not allow us to distinguish between private and professional internet use. While internet use for professional purposes is, therefore, included in the data for those who use their private device for work, this is not the case for those who have separate devices for work. This is particularly true for desktop or laptop devices. There are probably also differences in terms of how likely one is to use a private device for work based on their employer. Additionally, in the survey question, work use was explicitly included in the measure for overall usage time, whereas many participants in the tracking sample likely had additional devices at work that could not be tracked. This may explain parts of this 
overestimation of internet usage time, which is also in line with existing research (e.g., Araujo et al., 2017; Naab et al., 2019; Scharkow, 2016).

Moreover, our results indicate only small differences between age groups. While we did find differences in adoption rates of specific services, there were generally only small differences in usage time between age groups among the users of a service. In some instances, age differences were larger: for example, in the youngest age group (16-29), male internet users spent more than double the time on WhatsApp as compared to female users.

Also, the participants in all social groups spent the majority of their online time on a mobile device and, for instance, only one in ten visits to the online newspaper 20 Minuten was through a desktop device. It remains an open question whether this mobile-desktop ratio is different for other types of news outlets. However, the predominance of mobile over desktop internet use emphasizes the importance of tracking internet use on mobile devices including apps (previous studies mainly relied on browser plug-ins or tracking software for desktop devices, see e.g., Möller et al., 2019; Scharkow, 2016). The results regarding time of day showed that internet use started to increase in the early hours of the morning, peaked in the later afternoon and decreased thereafter.

A major advantage of the tracking method in this study was that it gathered observational data in a natural internet use situation. Effects of the measurement on participants' behavior were likely small, because after the initial installation, the tracking software did not interfere with participants' everyday internet use. Such an approach allows a more accurate approximation of their internet use. There are, however, still a number of limitations to consider. For research ethical reasons, it was technically possible for participants to temporarily disable tracking at any time-however, we assume social 
desirability effects are small because the data include widespread use of typically sensitive activities such as pornographic video consumption (1.4\% of all tracked events) ${ }^{2}$

Furthermore, there were respondents in the sample who reported using a service, but their tracking data did not include any tracked instances of that service. It is unclear whether this inconsistency can be attributed to the fact that the participants use these services only very rarely and did not happen to use them in the duration of the tracking data collection.

A few methodological conclusions for further studies relying on tracking data can be drawn from the empirical part of this article. Gathering and analyzing tracking-data is resource-intensive in many ways and entails specific challenges. Conducting a tracking study incurs high cost, particularly compared with collecting survey data. This is especially the case when the tracking data is collected over a long time span, for a representative sample and for multiple devices. As becomes apparent from the slight difference in representativeness between the tracking and survey sample in this study (see p. 6), motivating participants to participate in tracking studies in order to gather representative data remains a challenge.

Both the data collection and analysis processes tend to be more complex for tracking than for survey data including questions with closed answer categories. The tracking data that was collected for this study required significantly more cleaning and preprocessing prior to substantive analyses than the survey data. Whereas measuring what is supposed to be measured (e.g., time spent on an app) may be more error-prone in survey data (e.g., due to self-report biases), analyzing what is supposed to be analyzed may be more error-prone in tracking data. That is, were tracking completely unbiased, standard methods training and statistical software would still present practical obstacles to testing

\footnotetext{
${ }^{2}$ We calculated this figure by searching the tracking data for strings of the seven most popular pornographic video sites (see https:/www.menshealth.com/sex-women/g22481925/most-popular-pornsites/).
} 
communication theories with this relatively new source of data. In our case, having no predefined answer categories, a wide variety of services used and different names for services depending on language settings or the devices used in the tracking data made it challenging to ensure finding all occurrences of a specific service; and there is no standard for doing this yet. It is clear, however, that especially considering these biases, it is extremely important to make the code used for preprocessing and analyzing tracking data openly available.

Taking these challenges and limitations into account, tracking data-especially in combination with self-reported survey data-provides a promising empirical basis for answering various questions about digital media use and consequences in the future, especially when considering that any empirical data is a selective and incomplete depiction of reality. In line with emerging studies in the field (Mangold et al., 2021), our results indicate that generational and social gaps in internet usage time are relatively low and that this very basic question on internet usage differences still requires further research. This article has provided a quantitative description of internet usage time overall and of popular services across devices and social groups. In doing so, we highlighted advantages and challenges of measuring usage time with tracking data.

\section{Funding}

This project received funding from the Swiss National Science Foundation (SNF, Grant Nr. 176443).

\section{References}

Araujo, T., Wonneberger, A., Neijens, P., \& de Vreese, C. (2017). How much time do you spend online? Understanding and improving the accuracy of self-reported measures of internet use. Communication Methods and Measures, 11(3), 173190. https://doi.org/10.1080/19312458.2017.1317337

Boerman, S. C., Kruikemeier, S., \& Zuiderveen Borgesius, F. J. (2018). Exploring 
motivations for online privacy protection behavior: Insights from panel data. Communication Research. Online first. https://doi.org/10.1177/0093650218800915

Cardenal, A. S., Aguilar-Paredes, C., Cristancho, C., \& Majó-Vázquez, S. (2019). Echochambers in online news consumption: Evidence from survey and navigation data in Spain. European Journal of Communication, 34(4), 360-376. https://doi.org/10.1177/0267323119844409

Dubois, E., \& Blank, G. (2018). The echo chamber is overstated: The moderating effect of political interest and diverse media. Information, Communication \& Society, 21(5), 729-745. https://doi.org/10.1080/1369118X.2018.1428656

Dvir-Gvirsman, S., Tsfati, Y., \& Menchen-Trevino, E. (2016). The extent and nature of ideological selective exposure online: Combining survey responses with actual web log data from the 2013 Israeli Elections. New Media \& Society, 18(5), 857877. https://doi.org/10.1177/1461444814549041

Ernala, S. K., Burke, M., Leavitt, A., \& Ellison, N. B. (2020). How well do people report time spent on Facebook? An evaluation of established survey questions with recommendations. Proceedings of the 2020 CHI Conference on Human Factors in Computing Systems, 1-14. https://doi.org/10.1145/3313831.3376435

Guess, A., Nagler, J., \& Tucker, J. (2019). Less than you think: Prevalence and predictors of fake news dissemination on Facebook. Science Advances, 5(1). https://doi.org/10.1126/sciadv.aau4586

Jones-Jang, S. M., Heo, Y.-J., McKeever, R., Kim, J.-H., Moscowitz, L., \& Moscowitz, D. (2020). Good news! Communication findings may be underestimated: Comparing effect sizes with self-reported and logged smartphone use data. Journal of Computer-Mediated Communication, 25(5), 346-363. https://doi.org/10.1093/jcmc/zmaa009

Jürgens, P., Stark, B., \& Magin, M. (2019). Two half-truths make a whole? On bias in self-reports and tracking data. Social Science Computer Review, 38(5), 600-615. https://doi.org/10.1177/0894439319831643

Latzer, M., Büchi, M., \& Festic, N. (2020). Internet Use in Switzerland 2011-2019: Trends, Attitudes and Effects (Summary Report from the World Internet Project Switzerland). University of Zurich. https://mediachange.ch/research/wip-ch-2019/ Loecherbach, F., \& Trilling, D. (2020). 3bij3 - Developing a framework for researching 
recommender systems and their effects. Computational Communication Research, 2(1), 53-79. https://computationalcommunication.org/ccr/article/view/11

Mangold, F., Stier, S., Breuer, J., \& Scharkow, M. (2021). The overstated generational gap in online news use? A consolidated infrastructural perspective. New Media \& Society. Online first. https://doi.org/10.1177/1461444821989972

Möller, J., van de Velde, R. N., Merten, L., \& Puschmann, C. (2019). Explaining online news engagement based on browsing behavior: Creatures of habit? Social Science Computer Review, 38(5), 616-632. https://doi.org/10.1177/0894439319828012

Naab, T. K., Karnowski, V., \& Schlütz, D. (2019). Reporting mobile social media use: How survey and experience sampling measures differ. Communication Methods and Measures, 13(2), 126-147. https://doi.org/10.1080/19312458.2018.1555799

Scharkow, M. (2016). The accuracy of self-reported internet use-A validation study using client log data. Communication Methods and Measures, 10(1), 13-27. https://doi.org/10.1080/19312458.2015.1118446

Scharkow, M. (2019). The reliability and temporal stability of self-reported media exposure: A meta-analysis. Communication Methods and Measures, 13(3), 198211. https://doi.org/10.1080/19312458.2019.1594742

Smahel, D., MacHackova, H., Mascheroni, G., Dedkova, L., Staksrud, E., Olafsson, K., Livingstone, S., \& Hasebrink, U. (2020). EU Kids Online 2020: Survey results from 19 countries. London School of Economics and Political Science. http://www.lse.ac.uk/media-and-communications/research/research-projects/eukids-online

Stier, S., Breuer, J., Siegers, P., \& Thorson, K. (2019). Integrating survey data and digital trace data: Key issues in developing an emerging field. Social Science Computer Review, 38(5), 503-516. https://doi.org/10.1177/0894439319843669

Vraga, E. K., \& Tully, M. (2020). Who is exposed to news? It depends on how you measure: Examining self-reported versus behavioral news exposure measures. Social Science Computer Review, 38(5), 550-566. https://doi.org/10.1177/0894439318812050 